\title{
Revisión de programas de resiliencia basados en la evidencia en los ejércitos
}

\author{
García Silgo M. ${ }^{1}$
}

Sanid. mil. 2013; 69 (3): 182-194; ISSN: 1887-8571

\begin{abstract}
RESUMEN
Introducción: Los riesgos físicos y psicológicos asociados a las operaciones, el desarrollo del modelo positivo de salud mental y el avance en las prácticas basadas en la evidencia en psicología, han determinado que las estrategias de Defensa en determinados países incorporen programas de entrenamiento de la resiliencia para los militares y sus familias. Objetivos: Identificar tendencias en el estudio de la resiliencia en contextos militares; analizar el contenido de los programas de resiliencia en población militar; valorar las evidencias de dichos programas, entre ellos el Comprehensive Soldier and Family Fitness y el Battlemind Trainig; y examinar qué barreras dificultan la implementación de dichos programas en contextos militares. Método: Se emplearon bases de datos civiles y militares para realizar la revisión de publicaciones sobre resiliencia en personal militar. Se seleccionaron aquellas revisiones sistemáticas, metanálisis e investigaciones que hubieran demostrado un grado de evidencia fuerte o moderado. Resultados: Se observa una tendencia creciente a abordar la resiliencia en contextos militares desde las prácticas basadas en la evidencia; se demuestra que los contenidos de los programas militares de resiliencia se basan en evidencias fuertes o moderadas; se expone un resumen de programas militares de resiliencia; se detallan las fuertes evidencias en las que se basan el Battlemind Training y el Comprehensive Soldier and Family Fitness. y se relatan algunas de las barreras que obstaculizan la implementación de dichos programas en los ejércitos. Discusión: El avance de la psicología basada en la evidencia ha sido significativo en los ejércitos, redundando en resultados positivos en el personal militar. Sin embargo, existen todavía algunas barreras que dificultan realizar estos programas en contextos militares.
\end{abstract}

PALABRAS CLAVE: Resiliencia, estrés, salud ocupacional, preparación psicológica, Comprehensive Soldier and Family Fitness, Battlemind Training.

\section{A review of evidence-based resilience programs in the Army}

SUMMARY: Background: Since the end of the Cold War and the beginning of the asymmetric warfare, some countries are implementing resilience programs for the mental health's and well being of their military and families, shaped as Defense strategies. This has been possible because of the development of the positive mental health model and the evidence-based practice in psychology. Objectives: To summarize the information about resilience in the military; to review outstanding factors in resilience military programs; to show how resilience programs are already implemented; to analyze resilience evidenced-based main topics in the Comprehensive Soldier and Family Fitness and in the Battlemind Trainig; to help overcome the idiomatic hurdle; to analyze the barriers of resilience programs; and to suggest research topics. Methods: The publications about resilience and military were review in PsycINFO and DTIC. Meta-analysis, systematic reviews and randomized controlled trial with strong or moderate evidence were selected. Results: There is a growing trend in the use of evidence-based practices in military resilience programs. The content of the resilience programs is usually evidence-based. However, some of these programs haven't been validated yet. Comprehensive Soldier and Family Fitness and the Battlemind Training are described because of their good validation; finally, the barriers in the implementation of the programs are discussed. Discussion: The growing evidence-based psychology has been significant in military contexts, throwing positive results in the personnel. However, there are still some barriers to develop these programs in the Army.

KEY WORDS: Resilience, Stress, Occupational health, Mental health training, Comprehensive Soldier and Family Fitness, Battlemind Training.

\section{INTRODUCCIÓN}

Se entiende por resiliencia, la capacidad humana de asumir con flexibilidad situaciones límite y sobreponerse a ellas

${ }^{1}$ Cap. Psicólogo. Inspección General de Sanidad de la Defensa. Unidad de Apoyo Psicológico. Madrid. España.

Dirección para correspondencia: Mónica García Silgo. Unidad de Apoyo Psicológico. Inspección General de Sanidad de la Defensa. Glorieta del Ejército s/n. 28047 Madrid. España. Tlf: 914222968. mgarsil@et.mde.es

Recibido: 31 de enero de 2013

Aceptado: 23 de mayo de 2013
(Real Academia de la Lengua Española, $22^{\circ}$ ed.). Para Grotberg $^{1}$ es la habilidad para hacer frente a las adversidades de la vida, aprender de ellas, superarlas e incluso, ser transformados por estas (para una ampliación ver $\operatorname{citas}^{2,3}$ ). Como argumentan Southwick et al. ${ }^{4}$ la resiliencia se puede conceptualizar bien como resultado o respuesta al estrés, bien como proceso que media entre la respuesta al estrés y el trauma. Para Masten ${ }^{5}$ el fenómeno de la resiliencia, común entre personas que se enfrentan a experiencias traumáticas, surge de funciones y procesos adaptativos normales del ser humano. Además, según Manciaux, Vanistendael, Lecomte y Cyrulnik $^{6}$ la resiliencia es el resultado de un proceso dinámico y 


\section{Revisión de programas de resiliencia basados en la evidencia en los ejércitos}

evolutivo que varía según las circunstancias, la naturaleza del trauma, el contexto y la etapa de la vida y, además, se puede aprender, desarrollar o perfeccionar. Otros autores señalan que la naturaleza de la resiliencia es multidimensional ${ }^{7}$, por lo que, cuando una persona se enfrenta ante la adversidad puede ser muy competente en unas áreas pero no en otras. Según Southwick et al. ${ }^{3}$ la resiliencia es más dinámica que estática y no es un constructo dicotómico. Es decir, esta capacidad puede variar inter-situacionalmente e inter-temporalmente, por lo que un sujeto puede ser muy resiliente en unas situaciones pero no en otras, o serlo en un momento dado de su vida pero no en otro ${ }^{6}$.

Distintos autores ${ }^{2,7}$ han revisado numerosas definiciones de resiliencia y las han clasificado en tres grupos: como proceso o capacidad básica de sostenerse o resistir; como capacidad de recuperación, adaptación y vuelta al equilibrio inicial; y como crecimiento tras un acontecimiento traumático.

Se ha demostrado que la resiliencia protege de la depresión y facilita un mejor ajuste y adaptación ${ }^{8-10}$ y que los programas específicos de resiliencia muestran efectos positivos a largo plazo $^{10}$.

La literatura científica ha elaborado distintos modelos de resiliencia (ver tabla 1; para una revisión ver cita ${ }^{12}$ ). Los modelos centrados en las variables (variable-focused) buscan factores de protección específicos. Entre estos, se encuentran los modelos aditivos o de los efectos principales, los modelos indirectos o de efectos moduladores y los modelos interactivos o de efectos mediadores. Por otra parte, están los modelos centrados en las personas (person-focused) que son los que tratan de identificar personas resilientes y explicar en qué se diferencian de las que no lo son. Entre éstos destacan los modelos de caso único, de grupos y de diagnóstico completo (full diagnostic). Además, existen otros dos modelos. Los modelos que describen la trayectoria o el camino de las personas (pathway model) y los modelos transaccionales. Los modelos pathway son complicados de analizar debido a que la vida se va desplegando a través de numerosas interacciones, entre distintos sistemas y de maneras diversas ${ }^{12}$. Por último, los modelos transaccionales representan los riesgos psicosociales y los factores de protección en múltiples niveles de análisis (biológico, psicológico y social).

El reto, desde los modelos centrados en las variables, consiste en averiguar qué variables modulan y median la relación que hay entre los estresores y la salud. Entendiendo que: Los estresores o riesgos psicosociales son la interacción entre el contenido de trabajo, la gestión y organización del trabajo y las condiciones ambientales, por un lado, y las competencias y necesidades de los trabajadores, por otro ${ }^{13}$ (para una ampliación ver cita ${ }^{14)}$; que la salud es un estado de completo bienestar físico, mental y social, y no solamente la ausencia de afecciones o enfermedades ${ }^{15}$; que las variables moduladoras interactúan con los estresores de forma que disminuyen, aumentan o anulan los efectos de éstos sobre la salud ${ }^{14}$ y que las mediadoras explican la relación entre la predictora y la criterio $^{14}$.

\section{La resiliencia en los ejércitos}

La resiliencia ocupa un lugar relevante en los ejércitos de todos los tiempos ${ }^{16}$. Tradicionalmente, las estrategias militares universales de prevención psicológica han sido la selección de personal, el cribado, el entrenamiento, la cohesión y el liderazgo, pero ninguna de ellas incluía aportaciones del área de la salud mental ${ }^{17}$. El interés por la resiliencia por parte de líderes militares solía ser de tipo intuitivo (por ej. Clausewitz $\left.{ }^{18}\right)$. Con independencia de sus buenos resultados en muchas ocasiones, estas estrategias no han estado nunca contrastadas empíricamente. Sin embargo, en la actualidad, las aproximaciones a éste concepto están, cada vez más, basadas en la evidencia ${ }^{19,20}$.

Desde el fin de la guerra fría y el comienzo de los conflictos asimétricos, los programas de entrenamiento de la resiliencia del personal militar se han convertido en una prioridad para las estrategias de Defensa de algunos países ${ }^{21}$. En la última década, se han realizado diversos programas de entrenamiento de la resiliencia para militares, sus familias y los líderes de los ejércitos. Hay experiencias de dichos programas en las primeras fases del reclutamiento, en el ciclo del despliegue, en la preparación de los líderes, en el entrenamiento y preparación rutinaria de las unidades y en los momentos posteriores a un suceso traumático ${ }^{22}$. Aunque el programa Comprehensive Soldier and Family Fitness (CSF2; inicialmente conocido como Comprehensive Soldier Fitness, CSF) del ejército de los Estados Unidos, es el que ha recibido un mayor reconocimiento en publicaciones científicas y divulgativas, nacionales e internacionales, éste no es el único programa de este tipo, ni tampoco ha sido el primero. La

Tabla 1. Modelos de Resiliencia.

\begin{tabular}{|c|c|c|}
\hline $\begin{array}{l}\text { Modelos centrados en las } \\
\text { variables (variable-focused) }\end{array}$ & Buscan factores de protección específicos & $\begin{array}{l}\text { - Modelos aditivos o de efectos principales } \\
\text { - } \text { Modelos indirectos o de efectos moduladores } \\
\text { - } \text { Modelos interactivos o de efectos mediadores }\end{array}$ \\
\hline $\begin{array}{l}\text { Modelos centrados en las } \\
\text { personas (person-focused) }\end{array}$ & $\begin{array}{l}\text { Tratan de identificar personas resilientes y } \\
\text { de explicar en qué se diferencian de las que } \\
\text { no lo son }\end{array}$ & $\begin{array}{l}\text { - Modelos de caso único } \\
\text { - Modelos de grupos } \\
\text { - } \text { Modelos de diagnóstico completo (full diagnostic). }\end{array}$ \\
\hline $\begin{array}{l}\text { Modelos Mixtos } \\
\text { centrados en las variables y en las } \\
\text { personas }\end{array}$ & $\begin{array}{l}\text { Se centran en las transacciones y } \\
\text { mecanismos entre los sistemas }\end{array}$ & $\begin{array}{l}\text { - Modelos que describen la trayectoria o el camino de las } \\
\text { personas (pathway model) } \\
\text { - Modelos transaccionales }\end{array}$ \\
\hline
\end{tabular}


RAND Corporation detectó, en una revisión sistemática, 77 programas de resiliencia desarrollados en entornos civiles y de Defensa de distintos países: Estados Unidos, Canadá, Reino Unido, Australia e Israel ${ }^{7}$.

Los esfuerzos de la psicología militar por basar sus prácticas en la evidencia y, por tanto, trabajar desde la calidad y la excelencia, se plasman en numerosos estudios realizados con muestras militares de distintos países. Algunos ejemplos son los siguientes: Canadá ${ }^{23-25}$, Australia ${ }^{26,27}$, Sudáfrica ${ }^{28}$, Reino Unido ${ }^{29,30}$, Holanda ${ }^{31-33}$, Nueva Zelanda ${ }^{34}$, Noruega ${ }^{35,63,37,38}$, República Checa ${ }^{39}$, Israel ${ }^{40-43}$, Estados Uni$\operatorname{dos}^{17,44-47}$ o España ${ }^{48,49}$.

La OTAN también trabaja desde este enfoque. En 2009 creó, a través de la Science and Technology Organization (STO), el panel 203 ó Mental Health Training (traducido literalmente como «entrenamiento en salud mental», aunque en la práctica se concibe más como «entrenamiento psicológico» o «preparación psicológica») dependiente del Human Factors and Medicine (HFM), formado por un grupo de expertos, cuyo objetivo principal es desarrollar programas de entrenamiento de salud mental basados en la evidencia, que apoyen y ayuden al personal militar a lo largo de su carrera y les prepare para las operaciones. Una de las cuestiones que se ha manifestado en el panel HFM-203 es que no todos los países basan los programas de resiliencia en pruebas. $\mathrm{Si}$ bien la mayoría de los países apoyan el contenido de dichos programas en evidencias empíricas, muy pocos han llegado a validarlos demostrando efectos significativos en investigaciones controladas.

Mientras los programas de resiliencia que se implementen en los ejércitos no tengan en cuenta las evidencias empíricas, no se estará trabajando desde las prácticas basadas en la evidencia y, por tanto, serán programas basados en criterios aleatorios, intuitivos o tradicionalmente empleados y puede que buenas ideas, pero no se sabrá si éstos tienen efectos positivos o negativos sobre la salud, el bienestar y el rendimiento o si, realmente, no reportan ningún tipo de efecto. Según Castro y Adler ${ }^{50}$ la metodología basada en la evidencia consiste, primero, en que el contenido de los programas se base en material que haya sido investigado y, segundo, que el entrenamiento se haya validado. Además, estos autores añaden que, para la validación, no basta con que los programas hayan demostrado cambios positivos en una muestra en estudios pre- post, sino que es necesario que haya tenido efectos significativos en estudios controlados con muestras aleatorias.

\section{Modelo de resiliencia en los ejércitos}

La necesidad de fomentar la resiliencia tanto a nivel interno como externo o psicosocial, se hizo incuestionable en los ejércitos desde que se declaró que el despliegue, por sí mismo, no es el principal causante de los problemas psicológicos ocurridos durante o después de éste (Mental Health Advisory Team; MHAT) ${ }^{51}$. Sino que son los problemas psicosociales asociados a los despliegues los que provocan el aumento de psicopatología y absentismo y la disminución del bienestar y la salud. Esto significa que hay variables -como por ejemplo el apoyo familiar- que modulan los resultados de los estresores sobre la salud.

De este modo, los modelos de resiliencia desarrollados para los ejércitos suelen contemplar un amplio y complejo compendio de variables que interactúan y afectan al individuo, o que son parte de él. Por ejemplo, el modelo militar de demandas- recursos propuesto por Bates, Bowles, Hammermeister, Stokes, Pinder, Moore, et al. ${ }^{52}$ basado en la teoría de la conservación de los recursos de Hobfoll ${ }^{53}$ así como en el modelo laboral de demandas- recursos de Demerouti, Bakker, Nachreiner, et al. ${ }^{54}$, destaca como agentes relevantes en el análisis de la resiliencia tanto las demandas, riesgos o estresores, como los recursos internos y externos del individuo. La presente revisión analizará las evidencias que hay acerca estos recursos.

\section{OBJETIVOS}

Los objetivos generales que se proponen abordar de forma inespecífica a lo largo de este documento son: Proporcionar y resumir información relativa a la resiliencia en contextos militares; identificar aspectos relevantes en los programas de resiliencia en personal militar; mostrar las evidencias disponibles en el área; contribuir a superar la barrera idiomática; y sugerir temas de investigación. Los objetivos específicos son: Identificar posibles tendencias en el estudio de la resiliencia en contextos militares; analizar el contenido de los programas de resiliencia en población militar; valorar la validación de algunos programas como el CSF2 y el Battlemind Training; y examinar qué barreras dificultan la implementación de dichos programas en contextos militares.

\section{MATERIAL Y MÉTODOS}

Se realizaron búsquedas en organismos oficiales civiles (American Psychological Association; Ministerio de Sanidad, Servicios Sociales e Igualdad) y militares (Defense Technical Information Center -DTIC -; Science and Technology Organization -NATO-; RAND Corporation; Publicaciones de Defensa) y en la comunidad científica (PsycINFO, PsycARTICLES, Psicodoc y PubMed) con claves para la Biblioteca de la Universidad Complutense de Madrid. Las fuentes principales de búsqueda fueron PsycINFO y DTIC.

La selección de publicaciones se hizo en base a la fuente y al contenido, descartando las publicaciones desactualizadas, sin impacto ni prestigio, centradas en los aspectos negativos de la salud mental o que no estuvieran bien documentadas y se seleccionaron las revisiones sistemáticas y las investigaciones que hubieran demostrado un grado de evidencia fuerte o moderado siguiendo la clasificación de Meredith et al. (2011) 7 . Por motivos presupuestarios fueron descartados los artículos que supusieran un incremento del coste de la investigación. Sin embargo, si se destinó una partida a la compra de libros nacionales y extranjeros. 


\section{Revisión de programas de resiliencia basados en la evidencia en los ejércitos}

\section{Indice de impacto y de prestigio}

El índice de impacto se buscó en SCImago Journal and Country Rank ${ }^{55}$. El impedimento de no contar con índice de impacto para las publicaciones de editoriales - pese a la ya anunciada Book Publishers Citation Reports de Thomson Reuters-, se solventó mediante el índice de prestigio de la Scholarly Publishers Indicators (SPI ${ }^{56}$ ). El índice de impacto medio de las revistas de los artículos revisados para la introducción teórica es de 1.67 y de 1.48 para la revisión. El prestigio medio de las editoriales de los capítulos empleados en la introducción está en 155.36 y en 60.47 para la revisión. No se encontraron índices de prestigio para algunas editoriales como Brandeis University, M.E. Sharpe, Churchill Livingstone, Harvard Business School Press, Lyle Stuart, Lawrence Erlbaum Associates y la Esfera de los Libros. No obstante, la información aportada por estos documentos no se empleó como única fuente empírica y los autores contaban con una trayectoria reconocida en el área.

\section{RESULTADOS}

Empleando los descriptores «resilience« OR »psychological fitness « OR »mental strength « como palabras clave en PsycINFO, se encontraron 8838 registros. Para restringir la búsqueda a contextos militares, se añadieron los descriptores «military» OR «soldier» a la primera, conectados por el operador AND, localizando 295 publicaciones (ver Figura 1). De éstas, 223 eran artículos de revistas científicas, 58 eran libros y 14 eran tesis doctorales. En cuanto a la metodología, 175 eran estudios empíricos, 151 estudios cuantitativos, 27 estudios longitudinales, 25 entrevistas y 14 estudios prospectivos. Siguiendo con la descripción de la metodología basada en la evidencia de Castro y Adler ${ }^{50}$, se realizó una tercera búsqueda añadiendo el descriptor «randomized controlled trial» a la última, de la que surgieron 40 publicaciones, todas ellas realizadas en revistas científicas y, de las cuales, 4 pertenecían al área de «salud y servicios de salud mental», 6 al área de «psicofarmacología», 6 al de «trastornos de ansiedad y neuro-

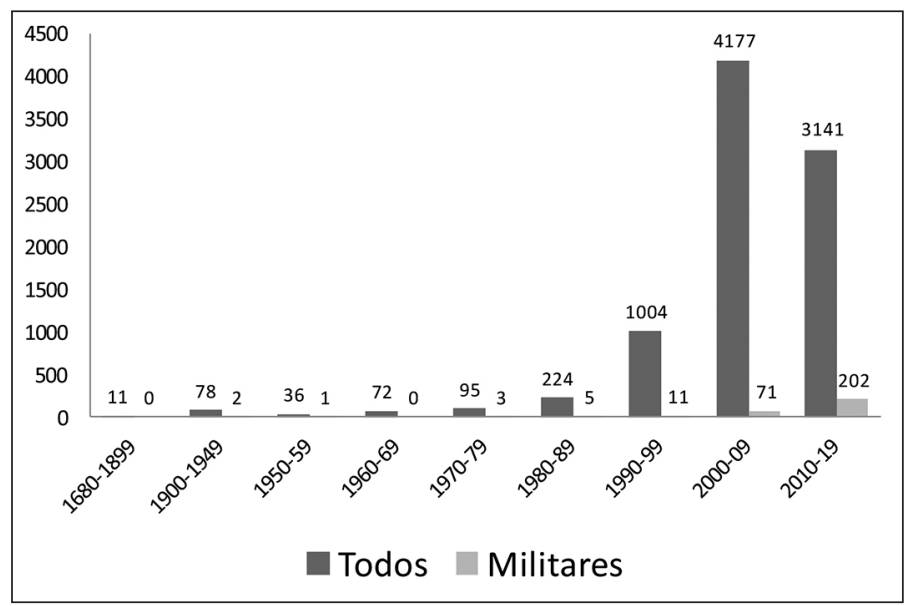

Figura 1. Evolución de las publicaciones sobre resiliencia en la comunidad cientifica. Fuente: PsycINFO (03/07/2013). sis» y 7 al de «terapias cognitivas». En la base de datos DTIC se encontraron 333 publicaciones. Del análisis de documentos reportados por PsycINFO y DTIC, se seleccionaron 60 documentos para hacer la revisión y 145 para hacer el artículo.

\section{Tendencias en las publicaciones científicas sobre resiliencia en contextos militares}

Se observa un crecimiento progresivo del número de publicaciones en la comunidad científica sobre resiliencia tanto en contextos civiles como militares (ver Figura 1). Ésta tendencia empieza a pronunciarse en la década de 1960-69 (con 72 publicaciones), acuciándose en la década de los 90 (1004 publicaciones). Pero es a partir de 2001 cuando se produce el gran auge de la resiliencia en la comunidad científica debido, según algunos autores como Meredith et al. $(2011)^{7}$, al atentado contra las Torres Gemelas, unido al comienzo formal de la Psicología Positiva tres años antes. Hay que destacar que en tres años y medio de la segunda década del siglo XXI se han publicado casi tantos documentos (3.141) como en toda la primera década del mismo siglo (4.177).

El contenido de las publicaciones militares sobre resiliencia, se puede subdividir en varias temáticas. Destacan las investigaciones con intervenciones que fomentan la resiliencia directamente en el personal militar $(46 \%)$ o en veteranos $(30 \%)$; otras lo hacen en las familias de los militares $(17 \%)$; y otras se centran en la promoción de la resiliencia de las unidades a través de los líderes $(9 \%)$. Las fases en las que se ha planteado la promoción de la resiliencia se pueden clasificar en: La Instrucción Básica de Combate, las Academias militares, la Instrucción y Adiestramiento, los programas de formación, la preparación para las operaciones, el Ciclo del Despliegue, la fase post-despliegue, tras acontecimientos traumáticos y en el entrenamiento de situaciones concretas de cursos específicos -como por ejemplo ser tomado prisionero-.

\section{Factores de resiliencia incluidos en los programas militares de entrenamiento psicológico}

Los programas que se están implementando en los ejércitos pretenden fomentar los factores de protección. Estos recursos internos y externos al sujeto, modulan la relación -o median- entre los estresores y la salud/enfermedad. El conjunto de todos los recursos positivos, formarían la resiliencia del individuo ante los riesgos. De tal modo que los militares con más recursos estarían más protegidos ante los riesgos físicos y psicosociales asociados a su profesión. En la tabla 2 se muestra la revisión realizada sobre las dimensiones internas de resiliencia que han demostrado alguna evidencia significativa y que se suelen incluir en los programas militares. La tabla 3 muestra la revisión de factores psicosociales o externos. Se seleccionaron estudios que hubieran demostrado fuertes evidencias en estudios controlados con muestras aleatorias y evidencias moderadas en estudios pre-post, en revisiones sistemáticas o metanálisis. Los factores marcados con asterisco 
Tabla 2. Evidencias de los factores internos incluidos en los programas de resiliencia.

\begin{tabular}{|c|c|c|}
\hline Factor interno & Evidencias & Revisado \\
\hline $\begin{array}{l}\text { Afecto positivo*: Es un constructo multidimensional que hace referencia a la capacidad de tener una actitud } \\
\text { optimista. Se compone de emociones positivas, actitud positiva, entusiasmo, vitalidad, optimismo, sentido del humor } \\
\text { en situaciones difíciles, esperanza, flexibilidad ante los cambios. }\end{array}$ & $57-65$ & 7 \\
\hline $\begin{array}{l}\text { Pensamiento Positivo*: Se compone de múltiples capacidades cognitivas como dar sentido a las situaciones, creatividad, } \\
\text { flexibilidad, aceptación, tener expectativas positivas sobre los resultados, procesamiento de la información aplicando } \\
\text { el conocimiento y permitiendo la restructuración, revaluación y reconfiguración positivas. Esta flexibilidad cognitiva, } \\
\text { ayuda en la toma de decisiones y en el afrontamiento positivo de los problemas. }\end{array}$ & $\begin{array}{l}58, \quad 59, \quad 62 \\
64,65,66,67\end{array}$ & 7,52 \\
\hline $\begin{array}{l}\text { Afrontamiento positivo*: Consiste en manejar las situaciones difíciles esforzándose para resolver situaciones } \\
\text { personales e interpersonales complicadas; buscar maneras de reducir o tolerar el estrés o el conflicto, incluyendo } \\
\text { aproximaciones activas, pragmáticas, espirituales, orientadas a la tarea, a la resolución o a la búsqueda de apoyo } \\
\text { social. }\end{array}$ & $65,68-70$ & 7,52 \\
\hline $\begin{array}{l}\text { Control del comportamiento*: Consiste en autoevaluar, autocontrolar y autopotenciar las reacciones emocionales para } \\
\text { conseguir un objetivo -autorregulación emocional- y requiere el desarrollo de habilidades para reconocer cambios físicos } \\
\text { y estados emocionales, así como regular eficazmente las conductas, las respuestas al estrés y las relaciones con los demás. } \\
\text { Una técnica empleada para desarrollar estas habilidades cognitivas de toma de conciencia (atención, orientación, alerta, } \\
\text { etc.) es el Mindfulness. }\end{array}$ & $71-76$ & 7,52 \\
\hline $\begin{array}{l}\text { Realismo*: Se relaciona con la capacidad de ser objetivo, de tener expectativas realistas sobre los resultados, confianza, } \\
\text { amor propio, autoestima, autoconcepto, autoeficacia y de aceptación de lo que se puede controlar y lo que no. }\end{array}$ & $73,76-83$ & 7 \\
\hline $\begin{array}{l}\text { Hardiness*: Es un constructo compuesto por tres dimensiones: compromiso, control y reto. La primera es el grado } \\
\text { en el que una persona se responsabiliza con la vida, su trabajo, su familia o su entorno, beneficia porque da sentido } \\
\text { a la vida. El segundo es el grado en el que uno percibe que controla lo que le ocurre en la vida, beneficia porque uno } \\
\text { siente que maneja su vida y que puede anticiparse a la adversidad. La tercera, consiste en percibir las amenazas como } \\
\text { oportunidades de crecimiento y aprendizaje, lo que facilita la adaptación. }\end{array}$ & $77,84-86$ & 52,87 \\
\hline $\begin{array}{l}\text { Autoeficacia*: Se define como los juicios que cada individuo tiene sobre sus capacidades, en base a los cuales } \\
\text { organizará y ejecutará sus actos de modo que le permitan alcanzar el rendimiento deseado. Consiste en la convicción } \\
\text { personal de que uno puede realizar con éxito cierta conducta requerida en una situación dada. }\end{array}$ & 88,89 & 52 \\
\hline $\begin{array}{l}\text { Espiritualidad: Se concibe como la capacidad para descubrir la esencia de uno mismo y las cuestiones sagradas en } \\
\text { la vida de uno. La espiritualidad se puede entender como estrategia de afrontamiento o como dimensión. En los } \\
\text { programas de resiliencia se integra tanto desde un punto de vista religioso como desde un punto de vista trascendental } \\
\text { y místico. }\end{array}$ & $90-96$ & 97,98 \\
\hline $\begin{array}{l}\text { Altruismo: Consiste en procurar el bien ajeno aún a costa del propio. Es un constructo que requiere del } \\
\text { individuo empatía, compasión y amabilidad, motivadas intrínsecamente. Aunque este factor ha sido propuesto } \\
\text { en distintos estudios, no hay fuertes evidencias a favor de él. Hecho que puede deberse a su escasa inclusión en } \\
\text { investigaciones. }\end{array}$ & 58 & 7 \\
\hline $\begin{array}{l}\text { Fortaleza física: Buena salud física. Funcionamiento eficiente y eficaz del cuerpo. Está relacionada con una buena } \\
\text { alimentación, ejercicio físico y vulnerabilidad genética. }\end{array}$ & $\begin{array}{l}75,77,99, \\
100\end{array}$ & 7 \\
\hline $\begin{array}{l}\text { Entrenamiento al estrés*: Consiste en la administración controlada, progresiva y continuada de estresores, de tal } \\
\text { forma que el sujeto se va adaptando poco a poco a la adversidad. Si a esto se le añade un entrenamiento en factores } \\
\text { positivos de resiliencia, el sujeto incrementa sus estrategias de afrontamiento ante la situación adversa a la que se va } \\
\text { a enfrentar. }\end{array}$ & 101-106 & \\
\hline $\begin{array}{l}\text { Experiencia laboral: Se asocia a una mayor habilidad para gestionar las tensiones propias del trabajo. Se relaciona } \\
\text { con una mayor edad. }\end{array}$ & 107 & \\
\hline
\end{tabular}

(*) se apoyan en evidencias fuertes, en resto lo hacen en evidencias moderadas.

Los factores descritos en las tablas 2 y 3 correlacionan negativamente con los problemas adaptativos, la depresión o el estrés $^{7-10,116}$ y con los problemas asociados a estos trastornos, como el absentismo, la accidentalidad, la indisciplina, las conductas adictivas, los problemas de pareja, etc. Así mismo, son restaurativos, aumentando el bienestar y la salud y correlacionan positivamente con la satisfacción laboral, la seguridad en las prácticas, la realización personal, familiar y social y facilitan la adaptación postraumática? . 


\section{Revisión de programas de resiliencia basados en la evidencia en los ejércitos}

Tabla 3. Evidencias de los factores externos incluidos en los programas de resiliencia.

\begin{tabular}{|c|c|c|}
\hline Factor externo & Evidencias & Revisado \\
\hline $\begin{array}{l}\text { Apoyo familiar*: Consiste en sentir que el confort se puede conseguir y es proporcionado por otros, incluyendo el } \\
\text { emocional, tangible, instrumental, informacional y espiritual. }\end{array}$ & $79,108-110$ & 7 \\
\hline $\begin{array}{l}\text { Clima positivo de unidad*: Se relaciona con el liderazgo eficaz, con facilitar y promocionar interacciones entre los } \\
\text { miembros de la unidad, construir un sentimiento de orgullo y de apoyo para la misión, y dar un buen ejemplo de } \\
\text { conducta. }\end{array}$ & $44,111,112$ & \\
\hline $\begin{array}{l}\text { Cohesión: Es la habilidad de la unidad para ejecutar acciones combinadas, tener un sentimiento de unión y un } \\
\text { compromiso de todos en la misión. }\end{array}$ & $65,113,114$ & \\
\hline Trabajo en equipo: Requiere que los miembros del grupo estén coordinados y tengan flexibilidad. & 75 & \\
\hline $\begin{array}{l}\text { Pertenencia a la comunidad*: Se asocia con la integración en grupos, tener amistades, participar en organizaciones } \\
\text { espirituales, protocolos, ceremonias, actividades sociales, etc. }\end{array}$ & 115 & \\
\hline
\end{tabular}

Tabla 4. Ejemplos de programas militares de entrenamiento de la resiliencia (adaptado de Meredith et al., 201177).

\begin{tabular}{|c|c|c|c|}
\hline Autor / Programa & Contenido & Objetivo & Implementación \\
\hline $\begin{array}{l}\text { Navy, Marine Corps, USA: } \\
\text { Combat Operational Stress } \\
\text { Control (COSC, 2007)l } \\
\text { Operational Stress Control } \\
\text { and Readiness (OSCAR, } \\
\text { 1999) }\end{array}$ & $\begin{array}{l}\text { Psicológico, } \\
\text { fís i c o, } \\
\text { psicosocial y } \\
\text { espiritual }\end{array}$ & $\begin{array}{l}\text { Prevenir y fomentar la salud y el } \\
\text { bienestar de los marines, marineros } \\
\text { y sus familias. Apoyar a los líderes. }\end{array}$ & $\begin{array}{l}\text { El personal que va a ser desplegado más de } 90 \text { días recibe el } \\
\text { COSC, normalmente en aulas y en tres fases: a los } 60 \text { días } \\
\text { de estar en zona; } 60 \text { días antes de regresar; y entre } 60 \text { y } 120 \\
\text { días después del despliegue. Está orientado a los líderes. } \\
\text { También hay un módulo para familias. El OSCAR trata de } \\
\text { unir la cultura operativa con la salud mental incorporando } \\
\text { instructores en los niveles de tropa. }\end{array}$ \\
\hline $\begin{array}{l}\text { National Security Agency, } \\
\text { USA: } \\
\text { Employee Engagement } \\
\text { Program (2005) }\end{array}$ & $\begin{array}{l}\text { Psicológico, } \\
\text { fís i c o, } \\
\text { psicológico } \\
\text { y espiritual }\end{array}$ & $\begin{array}{l}\text { Entrenar estratégicamente a los } \\
\text { líderes para aumentar su capacidad } \\
\text { de trabajar bajo presión y mejorar } \\
\text { su capacidad de gastar y recuperar } \\
\text { energía eficaz y eficientemente. }\end{array}$ & $\begin{array}{l}\text { El entrenamiento se hace en aulas. Primero se forma a } \\
\text { los instructores (un psicólogo, un entrenador físico, un } \\
\text { psicólogo experto en conducta y un enfermero). Cada uno de } \\
\text { ellos participará } 2 \text { días y medio. Además, se dedican entre } 4-8 \\
\text { horas a contenidos básicos. A los } 6 \text { meses del entrenamiento } \\
\text { se hace una evaluación del programa. }\end{array}$ \\
\hline $\begin{array}{l}\text { Army, West Point, USA: } \\
\text { Army Center for Enhanced } \\
\text { Performance (1993) -se ha } \\
\text { integrado en el CSF2- }\end{array}$ & $\begin{array}{l}\text { Psicológico } \\
\text { y físico }\end{array}$ & $\begin{array}{l}\text { Potenciar a los militares y a } \\
\text { sus familias mediante el uso } \\
\text { del pensamiento adaptativo, } \\
\text { la agilidad y las habilidades de } \\
\text { autorregulación. }\end{array}$ & $\begin{array}{l}\text { Dura entre } 6-8 \text { horas. Se imparte para grupos de entre } \\
40-60 \text { personas. El entrenamiento de la autoconciencia y } \\
\text { autorregulación se realiza en } 8-12 \text { horas de entrenamiento } \\
\text { y } 8-12 \text { horas de ejercicios. Normalmente se recibe antes del } \\
\text { despliegue aunque a veces se ha realizado durante o después } \\
\text { de éste. }\end{array}$ \\
\hline $\begin{array}{l}\text { HeartMath: } \\
\text { Civiles, USA (1999); } \\
\text { militares, USA (2007) }\end{array}$ & $\begin{array}{l}\text { Psicológico } \\
\text { y físico }\end{array}$ & $\begin{array}{l}\text { Mediante técnicas de biofeedback } \\
\text { se entrena la capacidad de «entrar } \\
\text { en coherencia» y llegar a estados } \\
\text { óptimos cognitivos, emocionales y } \\
\text { fisiológicos. }\end{array}$ & $\begin{array}{l}\text { Normalmente lo recibe el personal militar que se enfrenta } \\
\text { a niveles elevados de estrés. El programa puede durar } \\
\text { desde } 2 \text { horas a } 3 \text { días. Además hay entre } 4-6 \text { sesiones } \\
\text { telefónicas de seguimiento de entre } 30-60 \text { minutos. Los } \\
\text { instructores pueden ser veteranos de guerra, instructores } \\
\text { o psicólogos, todos ellos previamente acreditados por } \\
\text { HeartMath. }\end{array}$ \\
\hline
\end{tabular}

\section{Revisión de programas militares de entrenamiento de la resiliencia}

Reflejar todos los programas militares de resiliencia disponibles en la actualidad es inviable en el presente documento. No obstante, con el objetivo de explicar, escuetamente, en qué consisten y cómo se implementan dichos programas, se ha considerado mostrar cuatro ejemplos (tabla 4) y una descripción más profunda de los principales (CSF2 y BT).

\section{Comparación de tres programas de resiliencia: Battlemind Training, Battlemind Debriefing y sesiones psicoeducativas}

El grupo de investigadores del ejército de los Estado Unidos formado por Adler et al. ${ }^{65}$ se plantearon la necesidad de analizar y comparar el impacto de tres tipos de intervenciones psicológicas en militares norteamericanos recién llegados de Zona de Operaciones (ZO) a Territorio Nacional (TN). La muestra inicial era de 2.297 soldados americanos que habían estado un año 
desplegados en Irak. Tras eliminar a los sujetos que no acabaron el entrenamiento o que no completaron los cuestionarios, la muestra final quedó reducida a 1.060 sujetos. Las intervenciones psicológicas comparadas fueron: sesiones psicoeducativas básicas de manejo del estrés; el Battlemind Debriefing (BD) que está institucionalizado en el curso de control del estrés de combate del ejército norteamericano ${ }^{117}$ y el Battlemind Training (BT) que se basa en descubrimientos del Walter Reed Army Institute of Research (WRAIR) ${ }^{118}$ sobre la cohesión grupal, la seguridad, las relaciones personales, las reacciones biopsicosociales normales al combate o el apoyo a la vuelta a casa tras la misión, entre otros. El BD y el BT se diferencian, fundamentalmente, en el grado de interacción de los miembros del grupo y en la orientación cognitiva.

El BT fue la primera iniciativa para entrenar la resiliencia en el ejército de los Estados Unidos y, por sus buenos resultados, el Secretario de Ejército de éste país lo integró en el Programa de Apoyo al Ciclo del Despliegue ${ }^{119}$. Según Castro, Hoge y Cox ${ }^{120}$ y Adler et al. ${ }^{65}$ el BT cuenta con módulos diferenciados para soldados, líderes, familias y reservistas; se imparte en grupos reducidos de personas, en un número determinado de sesiones, de 35-40 minutos cada una; y se desarrolla en dos fases de aplicación, una pre-despliegue y otra post-despliegue, esta última con dos subfases: la primera, en las dos primeras semanas tras el despliegue y la segunda a los 3-6 meses. Mediante la metodología interactiva, el BT contempla aspectos reales de los soldados; propone alternativas y soluciones prácticas; replantea positivamente y, mediante acciones concretas, las dificultades que se puedan presentar; destaca que las habilidades que pueden servir para adaptarse a $\mathrm{ZO}$ puede que no sean adaptativas en TN; y trabaja el estigma asociado a la búsqueda de ayuda por problemas psicológicos. Distintos estudios ${ }^{51}$ han reflejado que hay una relación significativa entre el BT en fases pre-despliegue y la salud mental de los soldados durante el despliegue y que los módulos post-despliegue son eficaces en diseños controlados.

Respecto a la traducción del término BT al castellano, hay que decir que la palabra anglosajona battlemind tiene una traducción al castellano compleja, además de interesante de cara a las operaciones. Motivo por el que se ha incluido la siguiente explicación. Battlemind es la unión de dos palabras battle (batalla) y mind (mente), por lo que battlemind training se podría traducir como «entrenamiento de la mente para la batalla». E1 término battlement (fortaleza, muralla) es también relevante para la traducción, teniendo en cuenta que las definiciones de battlemind han sido «fortaleza inherente al soldado para enfrentarse con coraje al miedo o a la adversidad en el combate» ${ }^{65}$, «fortaleza innata al soldado para afrontar, con confianza y determinación, la adversidad, el miedo y el sufrimiento durante el combate» ${ }^{120} \mathrm{y}$ 《el entrenamiento de la fortaleza del guerrero ante el peligro ${ }^{50}$. Por lo tanto, battlemind podría traducirse como fortaleza psicológica o resiliencia. Por otra parte, Adler et al. ${ }^{65}$ explican que la palabra battlemind se emplea en reconocimiento a los aspectos psicosociales en el combate como el compañerismo, el liderazgo, la autoconfianza, la reflexión ante los riesgos, el manejo de los retos futuros, el desarrollo de la dureza mental, la superación de los reveses cotidianos o el mantenimiento de pensamientos positivos en tiempos difíciles. Además, cada una de las 10 letras de la palabra battlemind corresponde con las 10 habilidades que los militares deben desarrollar durante el despliegue para adaptarse y que pueden causar problemas en fases post-despliegue si no consiguen readaptarse al volver ${ }^{17}$ :

- Buddies: Amistad y cohesión en ZO vs. Evitación, no integración y retraimiento en $\mathrm{TN}$

- Accountability: Responsabilidad en ZO vs. Control en TN

- Targeted Aggression: Agresión dirigida en ZO vs. Agresión inapropiada en TN

- Tactical Awareness: Conciencia táctica en ZO vs. Hipervigilancia en TN

- Lethally armed: Letalmente armado en ZO vs. «Cargado y encerrado» en TN

- Emotional Control: Control emocional en ZO vs. Desapego e ira en TN

- Mission Operational Security: Seguridad en la misión en $\mathrm{ZO}$ vs. Secretismo en TN

- Individual Responsibility: Responsabilidad individual en $\mathrm{ZO}$ vs. Culpa en TN

- Non-Defensive (Combat) Driving: Conducción de combate en $\mathrm{ZO}$ vs. Conducción temeraria en $\mathrm{TN}$

- Discipline and Ordering: Órdenes y disciplina en ZO vs. Conflicto y desorden en TN

Por otra parte, el BD se basa en la técnica del Debriefing Psicológico, empleada en salud mental. El BD utiliza recursos del debriefing efectuado por líderes militares tras las actuaciones (leader-led-after-action debriefing), del debriefing de Eventos Críticos (Critical Event debriefing) y de modelos de debriefing civiles como la Gestión de Estrés por Incidentes Críticos (Critical Incident Stress debriefing). Además, el BD se centra en el clima y la cohesión grupal mediante tres asuntos clave. El primero es que se reconoce que la transición entre estar desplegado en $\mathrm{ZO}$ y la vuelta a la rutina en TN es una tarea psicosocial crítica ${ }^{65}$; El segundo es que se refuerza la idea de que los eventos traumáticos relacionados con el despliegue ocurrieron en el pasado y no son parte de las experiencias cotidianas y, el tercero, es la promoción del apoyo entre compañeros y líderes.

El BD es una técnica controvertida debido a que algunos investigadores han encontrado que puede tener efectos dañinos en víctimas civiles de sucesos traumáticos ${ }^{121-124} \mathrm{o}$ no generar todos los resultados positivos que se esperaban en personal militar combatiente ${ }^{125}$. Sin embargo, puesto que la mayor parte de estas investigaciones presentan problemas de diseño metodológico o se han realizado con muestras pequeñas, Adler et al. ${ }^{65} \mathrm{se}$ plantearon ponerla a prueba en el experimento que se empezó a describir en este apartado y que continúa como sigue a continuación.

Entre julio y agosto de 2005, pocos días después de haber vuelto de Irak, el personal de la muestra participó en un programa de 7 días de readaptación. En estos días se estableció la línea base con un cuestionario que medía el grado de exposición al combate, el estigma asociado a la búsqueda de ayuda psicológica, las actitudes hacia el entrenamiento y la sintomatología de Trastorno de Estrés Postraumático (TEPT), depresión y trastornos del sueño. Cuatro meses después, la muestra volvió 


\section{Revisión de programas de resiliencia basados en la evidencia en los ejércitos}

a responder al mismo cuestionario. Tras la evaluación inicial se distribuyó al personal de manera aleatoria a cualquiera de estas cuatro condiciones experimentales:

- BD: 26 sesiones, de 50 minutos, en grupos de 20 a 32 personas $(\mathrm{n}=271)$

- BT1: 23 sesiones, de 39 minutos, en grupos de 18 a 45 sujetos $(\mathrm{n}=272)$

- BT2: 6 sesiones, de 39 minutos, en grupos de 126 a 225 sujetos $(n=274)$

- Psicoeducación sobre manejo del estrés: 5 sesiones, de 40 a 50 minutos, en grupos de 51 a 257 sujetos $(n=243)$

Los resultados mostraron que los sujetos experimentales que habían estado expuestos a elevados niveles de exposición al combate se beneficiaban tanto del BD como del BT. No hubo diferencias entre los grupos BD y BT1, ni entre BT1 y BT2. En concreto, se observó que los sujetos que formaron parte del grupo BD y del grupo BT1 y BT2, mostraban menos síntomas de TEPT y depresión que el grupo de psicoeducación. Respecto a los problemas con el sueño en militares con alta exposición al combate, el grupo que recibió el BD mostró menos sintomatología que el de psicoeducación. Por último, el grupo expuesto al combate que formó parte del grupo BT2, mostró menos estigma que el grupo de psicoeducación.

Por lo tanto, las dos intervenciones BD y BT mostraron ser más eficaces que el grupo de psicoeducación. Además, puesto que los resultados fueron positivos tanto en el grupo BT1 como BT2, se concluye que el tamaño de los grupos no explica los resultados. En la investigación se demuestra que el BT es una aproximación positiva de tipo cognitivo capaz de reducir, meses después del despliegue, los problemas de salud mental y el estigma asociado a estos.

\section{Evidencias en las que se basa el programa Comprehensive Soldier and Family Fitness (CSF2)}

El programa de resiliencia del ejército de los Estados Unidos CSF2 evalúa y entrena cinco factores de resiliencia de los militares -tres internos y dos psicosociales-: Emocional, espiritual, físico, social y familiar. E1 CSF2 aprovecha la psicología científica para fomentar la resiliencia de los soldados en un programa continuado de fortalecimiento ${ }^{19,20}$. El contenido del CSF2 está basado en cuatro evidencias empíricas: La primera es que se basa en el programa de resiliencia de la Universidad de Pensilvania -Penn Resiliency Project (PRP)- y éste se ha replicado en 17 experimentos controlados de prevención de ansiedad y depresión ${ }^{126}$, por lo que es el programa de resiliencia más replicado de la literatura científica ${ }^{127}$ habiéndose demostrado que mejora las habilidades cognitivas y sociales, reduce la ansiedad, la depresión, los trastornos adaptativos y los problemas de conducta ${ }^{128}$; la segunda es que el PRP es el único programa psicológico en el que el entrenamiento de los entrenadores se ha validado ${ }^{127}$; la tercera es que el PRP se ha replicado en una muestra militar ${ }^{70}$; y la cuarta es que parte del contenido del CSF2 es el programa Battlemind Training, por ser éste uno de los mayores logros en salud mental del WRAIR ${ }^{45}$. El nombre
Battlemind Training se sustituyó por Resilience Training para integrarse en el CSF2.

E1 programa CSF2 comienza cuando el sujeto realiza la prueba Global Assessment Tool (GAT; Herramienta de Evaluación Global), con la que se miden cuatro dimensiones de la salud ${ }^{129}$ : Familiar; espiritual; social -en el que se toman dos variables, la confianza y la amistad-; y emocional -en donde se incluyen las emociones positivas, negativas, la depresión, el optimismo, la flexibilidad, el compromiso con el trabajo, el afrontamiento activo de los problemas y algunas fortalezas del carácter como la sabiduría, valentía, humanidad, justicia, templanza y trascendencia-.

Puesto que se ha demostrado la importancia del contexto social en el bienestar de los soldados (por ej. Bliese ${ }^{44}$ ), se determinó, que para que el CSF2 fuera eficaz, habría que incluir en el GAT variables organizacionales, como la cohesión grupal y el liderazgo ${ }^{130}$. De este modo, a la primera versión de 16 subescalas del GAT, se añadieron otras dos, creando una versión extendida $(\mathrm{GAT}+)$. El GAT+ permitirá comparar en qué circunstancias organizacionales el CSF2 obtiene los resultados más eficaces. Los resultados de ambas pruebas se emplean para tomar la línea base de cada sujeto en cada dominio -nunca para fines diagnósticos- con el fin de fortalecer las áreas necesarias. Hay que destacar que el GAT es relevante porque ${ }^{131}$ : Es un inventario sistemático que mide la salud psicosocial de los soldados y los describe en términos multidimensionales; evalúa el bienestar no sólo por la ausencia de problemas, sino por la presencia de salud; introduce vocabulario común acerca de lo que está bien; proporciona retroalimentación de las fortalezas del soldado en el instante que realizan el test, ya que se ha demostrado que recibir información inmediata a la evaluación es un componente crítico para el cambio ${ }^{132}$; reduce el estigma asociado a los problemas mentales; y ofrece la posibilidad de conocer las fortalezas y debilidades que se deben abordar en la intervención posterior. Al proporcionar información individualizada, la eficacia de las intervenciones se incrementa notablemente ${ }^{133}$.

Otro de los pilares del CSF2 es el Master Resilience Trainer (MRT; Entrenador Experto en Resiliencia). El contenido del MRT se basa en el PRP y se organiza en un curso de 10 días, dirigido a suboficiales, donde aprenden estrategias resilientes para uno mismo y los demás ${ }^{128}$. También hay experiencias piloto del MRT en familias de militares. El PRP se basa en el modelo de prevención de ansiedad y depresión propuesto en el «Proyecto APEX» de Gillham et al. ${ }^{128}$ e incorpora algunos de los factores de protección descritos por Masten y $\operatorname{Redd}^{134} \mathrm{y}$ Masten et al. ${ }^{12}$ que contribuyen a crear resiliencia (optimismo, eficacia en la resolución de problemas, autoeficacia, flexibilidad, control de impulsos, empatía, conciencia emocional y relaciones interpersonales íntimas). El elemento central del PRP y del APEX es el modelo A-B-C de Ellis ${ }^{135}$ que se basa en que las creencias que uno tiene dirigen las emociones y las conductas. El último componente del CSF2 es el módulo de Crecimiento Postraumático (descrito por Silgo $^{16}$ ).

Un aspecto importante que ya se incluía en el Battlemind Training y se incorporó en el CSF2, es el reconocimiento al estigma asociado a los trastornos mentales. El estigma se ha definido como «un atributo profundamente deshonroso» ${ }^{136}$. Cuan- 
do un militar presenta un problema mental, no sólo debe hacer frente a los síntomas, a los estresores que lo originaron y a las consecuencias de los síntomas. Además debe afrontar el rechazo social por tener problemas mentales (estigma) y, a veces, el rechazo de uno mismo (autoestigma). El estigma puede ser un obstáculo para no buscar ayuda y por tanto provocar el agravamiento de los síntomas (para más información sobre estigma en militares ver $\left.\operatorname{cita}^{119}\right)$.

\section{Validación del Comprehensive Soldier and Family Fitness (CSF2)}

Resultados preliminares del CSF2 indican que hay una relación significativa entre conducta negativa (consumo de drogas ilícitas, cometer delitos y suicidarse) y baja resiliencia ${ }^{137}$ y conducta positiva (rendimiento laboral elevado) y alta resiliencia $^{138}$. También se ha señalado ${ }^{139}$ que los soldados cuyos suboficiales han recibido el MRT presentan niveles significativamente más altos de resiliencia y salud psicológica que los soldados destinados con suboficiales que no hicieron dicho curso; que no hay evidencias de que estos soldados «empeoren» su salud mental; que los cursos MRT más efectivos son los que se desarrollan en sesiones formales, cuando el Mando selecciona líderes de confianza para hacerlo y cuando el Mando apoya adecuadamente el desarrollo del MRT. Este informe también destaca que las habilidades que se entrenan en el MRT son significativamente más efectivas para los soldados de entre 18-24 años que para los que superan esta edad. Un cuarto informe ${ }^{140}$ realizado con 7230 militares, revela que la exposición al entrenamiento en resiliencia aumenta los factores de la resiliencia de los soldados, así como la salud psicológica y reduce la probabilidad de desarrollar un trastorno mental o problemas de abuso de sustancias.

\section{Barreras que impiden el desarrollo de programas de resiliencia en los ejércitos}

En la literatura científica es muy poco común analizar las barreras que obstaculizan la implementación de programas militares de resiliencia. No obstante, como señalan Lester et al. ${ }^{139}$, la efectividad de los programas no solo dependen del contenido, también juegan un papel relevante los instructores del programa o el apoyo al propio programa por parte de los líderes.

Para analizar las barreras con las que se encuentra el personal encargado de desarrollar los programas de resiliencia en contextos militares, Meredith et al. ${ }^{7}$ entrevistaron a 23 representantes de los programas de resiliencia más relevantes, recogiendo información sobre los distintos tipos de obstáculos. Entre las barreras relacionadas con la implementación de los programas, destacaron: la falta de financiación; no recibir apoyo por parte del Mando; y problemas logísticos como no mantener al personal, no coordinar las actividades o no proporcionar un espacio físico adecuado. También se evidenciaron barreras atribuidas al estigma asociado a los problemas mentales, a la falta de aceptación de estos programas en audiencias militares no clínicas y a la dificultad para realizar estos programas en fases pre-despliegue debido, fundamental- mente, a la exigente agenda a la que el personal es sometido en fases de preparación.

\section{DISCUSIÓN}

Se observa una tendencia creciente, en los programas militares de resiliencia, a basar las prácticas en la evidencia. Destacan las intervenciones que fomentan la resiliencia directamente en los militares, otras lo hacen en las familias de éstos y las terceras se centran en entrenar estilos de liderazgo que promueven la resiliencia de las unidades. Las fases en las que se están implementando los programas de resiliencia son: En la Instrucción Básica de Combate, en las Academias militares, en la Instrucción y Adiestramiento, en los programas de formación, en la preparación para operaciones, en el Ciclo del Despliegue, en la fase post-despliegue, tras acontecimientos traumáticos y en el entrenamiento de situaciones concretas de cursos específicos.

Los factores internos y externos de resiliencia que han recibido un mayor apoyo empírico han sido: Afecto positivo, pensamiento positivo, estrategias positivas de afrontamiento, control del comportamiento, realismo, hardiness, autoeficacia, espiritualidad, entrenamiento al estrés, altruismo, fortaleza física, experiencia laboral, apoyo familiar, clima positivo de unidad- liderazgo, cohesión grupal, trabajo en equipo y pertenencia a la comunidad.

Una de las medidas con más consistencia para superar las barreras que impiden implementar programas de resiliencia en contextos militares, es que los programas reciban un fuerte apoyo por parte del Mando ${ }^{7,119}$

Mientras las evidencias empíricas apoyan el uso de los factores de resiliencia descritos en este trabajo y muchos países los incluyen en sus programas, todavía no se ha generalizado la validación de éstos en los ejércitos de nuestro entorno. Tampoco se ha llegado a consensuar cuáles de éstos factores estarían en un nivel prioritario y cuáles en uno secundario o terciario. Priorizar los factores sería una tarea recomendable, teniendo en cuenta que estos programas se implementan en entornos laborales de riesgo en los que existen barreras que impiden su incorporación a los planes operativos, de enseñanza, formación o de Instrucción y Adiestramiento.

Pese a que se están implementando programas de resiliencia en todas las fases de la carrera del personal militar, algunas cuestiones habría que aclararlas. Por ejemplo, qué diferencias individuales, entre los militares que reciben estos programas, favorecen o dificultan el éxito de los mismos. Otra cuestión a analizar sería el papel que juegan los instructores que van a impartir los programas, qué características deben tener para que el programa sea eficaz, si deben ser expertos en salud mental o si basta con que reciban un buen entrenamiento en resiliencia para que puedan enseñarlo a otros. Además, habría que dilucidar si en todas las fases en las que se implementan estos programas se consiguen efectos similares en la prevención de la salud mental y el bienestar o si hay fases de la carrera militar en las que se consigue un mayor beneficio.

Por otra parte, puesto que no hay todavía protocolos de programas de resiliencia individualizados -desde que el sujeto ingresa a la organización, hasta que se va- podría ocurrir que se 


\section{Revisión de programas de resiliencia basados en la evidencia en los ejércitos}

sature al personal con estos programas a lo largo de su carrera y, por tanto, pierdan su razón de ser. Por este motivo, la labor de gestión y organización de los programas de resiliencia, parece necesaria.

No se ha tratado en el documento las diferencias culturales, pero en las referencias se observa que todas las evidencias proceden de estudios extranjeros, en su mayoría, del ejército de los Estados Unidos. En Europa, Noruega y Holanda cuentan con una mayor solidez. Teniendo en cuenta que las diferencias culturales pueden conllevar distintos resultados en los programas y que las estrategias de Defensa de los países de nuestro entorno ya contemplan los programas de resiliencia como una de las prioridades, se recomienda fomentar en España la investigación en este área.

\section{REFERENCIAS}

1. Grotberg, E.H. (2006). ¿Qué entendemos por resiliencia? ¿Cómo promoverla? ¿Cómo utilizarla?. En E.H. Grotberg (Ed.) La Resiliencia en el Mundo de Hoy: Cómo Superar Adversidades. Barcelona: Gedisa.

2. Reich, J.W., Zautra, A.J. y Hall, J.S. (Eds.). (2010). Handbook of Adult Resilience. New York: Guilford Press.

3. Southwick, S.M., Litz, B.T., Charney, D. y Friedman, M.J. (Eds.). (2011) Resilience and Mental Health: Challenges Across the Lifespan. Cambridge: Cambridge University Press.

4. Masten, A.S. (2001). Ordinary Magic: Resilience process in development American Psychologist, 56, 227-238.

5. Manciaux, M., Vanistendael, S., Lecomte, J. y Cyrulnik, B. (2005). La resiliencia: estado de la cuestión. En M. Manciaux (Ed.), La Resiliencia: Resistir y Rehacerse. Barcelona, Gedisa.

6. Masten, A.S., Moon, A.R. y Supkoff, L.M. (2011). Resilience in children and adolescents. En S.M. Southwick, B.T. Litz, D. Charney y M.J. Friedman (Eds.): Resilience and Mental Health: Challenges Across the Lifespan. Cambridge: Cambridge University Press.

7. Meredith, L.S., Sherbourne, C.D., Gaillot, S., Hansell, L., Ritschard, H.V., Parker, A.M. y Wrenn, G. (2011). Promoting psychological resilience in the U.S. military. Santa Monica, CA. RAND Corporation.

8. Fredrickson, B.L. y Tugade, MM. (2003). What Good are Positive Emotions in Crises? A Prospective Study of Resilience and Emotions Following the Terrorist Attacks on the USA on September 11 $11^{\text {th }}, 2001$. Journal of Personality and Social Psychology, 84, 365-376.

9. Peterson, C., Park, N. y Seligman, M.E.P. (2006). Greater strengths of character and recovery from illness. Journal of Positive Psychology, 1, 17-26.

10. Peterson, C., Ruch, W., Beermann, W., Park, N. y Seligman, M.E.P. (2007). Strengths of character, orientation to happiness, and life satisfaction. Journal of Positive Psychology, 2, 149-156.

11. Duckworth, A.L., Steen, T.A. y Seligman, M.E.P. (2005). Positive Psychology in Clinical Practice. Annual Review of Clinical Psychology. 1, 629-651.

12. Masten, A.S., Cutuli, J.J., Herbers, J.E. y Reed, M.G.J. (2009). Resilience in development. En S.J. Lopez y C.R. Snyder (Eds.). Oxford Handbook of Positive Psychology, ( $2^{\mathrm{a}}$ Ed). New York: Oxford University Press.

13. Organización Internacional del Trabajo (1986). Psychosocial Factors at Work: Recognition and Control. Occupational Safety and Health, 56. OIT.

14. González-Camino, G. y Osca, A. (2004). Estrés y salud laboral. En A. Osca (Ed.): Psicología de las Organizaciones (PP. 301-344). Madrid: Sanz y Torres.

15. Organización Mundial de la Salud (1948). Preámbulo de la Constitución de la Organización Mundial de la Salud, adoptada por la Conferencia Sanitaria Internacional, Nueva York, 19 de junio- 22 de julio de 1946, firmada el 22 de julio de 1946 y entró en vigor el 7 de abril de 1948.

16. Silgo, M.G. (2012a). El papel de la resiliencia en las Fuerzas Armadas. En D. Donoso Rodriguez (Coord.): Psicología en las Fuerzas Armadas. Ministerio de Defensa, Secretaria General Técnica. http://publicacionesoficiales. boe.es/
17. Nash, W.P., Krantz, L., Stein, N., Westphal, R.J. y Litz, B. (2011). Comprehensive soldier fitness, battlemind, and the stress continuum model: Military organizational approaches to prevention. En J.I. Ruzek, P.P. Schnurr, J.J. Vasterling y M.J. Friedman (Eds.): Caring for veterans with deployment-related stress disorders: Irak, Afganistan, and beyond, 193-214. Washington DC: American Psychological Association.

18. Clausewitz, C.V. (2005). De la Guerra. ( $1^{\text {a }}$ Ed). Versión íntegra. Madrid: La esfera de los libros.

19. Casey, G.W. (2011). Comprehensive Soldier Fitness: A vision for psychological resilience in the U.S. Army. American Psychologist, 66,1,1-3.

20. Cornum, R.L., Matthews, M.D y Seligman, M.E.P. (2011). Comprehensive Soldier Fitness: Building resilience in a challenging institutional context. American Psychologist, 66, 1, 4-9.

21. National Security Research Division. (2012). Annual Report 2011-2012. Santa Mónica, CA: RAND Corporation, National Defense Research Institution.

22. Silgo, M.G. (2012b). Intervenciones de resiliencia en contextos militares. En M.P. Bardera (Coord.): Riesgos Psicosociales y Resiliencia en Cuerpos y Fuerzas de Seguridad. Jornadas celebradas el 12/12/12. Madrid: UNED.

23. Farley, K.M.J. (2003). A model of unit climate and stress for Canadian soldiers on operations. Tesis doctoral número: AAINQ79455.

24. Lee, J.E.C., Sudom, K.A. y McCreary, D.R. (2011). Higher-Order model of resilience in the Canadian forces. Canadian Journal of Behavioural Science, $43,3,222-234$.

25. Skomorovsky, A. y Sudom, K.A. (2011). Psychological well-being of Canadian forces officer candidates: The unique roles of hardiness and personality. Military Medicine, 176, 4, 389-396.

26. McCormack, L. y Mellor, D. (2002). The role of personality in leadership: An application of the five-factor model in the Australian military. Military Psychology 14, 179-197.

27. Creamer, M., Wade, D., Fletcher, S. y Forber, D. (2011). PTSD among military personnel. International Review of Psychiatry, 23, 2, 160-165.

28. Van Breda, A.D. (2008). The military social health index: A partial multicultural validation. Military Medicine, 173, 5, 480-487.

29. Hardy, L., Arthur, C.A., Jones, G., Shariff, A., Munnoch, K. et al. (2010). The relationship between transformational leadership behaviors, psychological, and training outcomes in elite military recruits. The Leadership Quarterly, 21, 1, 20-32.

30. Brewin, C.R., Garnett, R. y Andrews, B. (2011). Trauma, identity and mental health in UK military veterans. Psychological Medicine, 41, 1733-1740.

31. Van den Berg, C.E. (2009). Soldiers under threat: An exploration of the effect of real threat on soldiers' perceptions, attitudes and morale. Tesis Doctoral. Nederlandse Defensie Academie, Faculteit Militaire Wetenschappen, Breda, Nederland

32. Schok, M.L., Kleber, R.J. y Lensvelt-Mulders, G.J.L.M. (2010). A model of resilience and meaning after military deployment: Personal resources in making sense of war and peacekeeping experiences, Aging and Mental Health, $14,3,328-338$.

33. Delahaij, R., Gaillard, A.W.K. y Van Dam, K. (2010). Hardiness and the response to stressful situations: Investigating mediating processes, Personality and Individual Differences, 49, 5, 386-390.

34. Gardner, D. y Carston, M.C.(2009). Cognitive hardiness in the New Zealand military. New Zealand Journal of Psychology, 38, 3, 26-34.

35. Eid, J. y Morgan, C.A. (2006). Dissociation, Hardiness and performance in military cadets participating in survival training. Military Medicine, 171, $5,436-442$

36. Eid, J., Johnsen, B.H., Bartone, P.T. y Nissestad, O.A. (2007). Growing transformational leaders: exploring the role of personality hardiness. Leadership \& Organization Development Journal, 29, 1, 4-23.

37. Hystad, S.W., Eid, J. y Brevik, J.I. (2011). Efects of psychological hardiness, job demands, and job control on sickness absence: A prospective study. Journal of Occupational Health Psychology, 16, 3, 265-278.

38. Hystad, S.W., Eid, J., Laberg, J.C. y Bartone, P.T. (2011). Psychological hardiness predicts admission into Norwegian military officer schools. Military Psychology, 23, 4, 381-389.

39. Krutis, J., Mares, J. y Jezek, S. (2011). Posttraumatický rozvoj u vojáku acr po návratu ze zahranicní mise. Ceskoslovenska Psychologie, 55, 3, 245-256.

40. Florian, V., Mikulincer, M. y Taubman, O. (1995). Does Hardiness contribute to mental health during a stressful real-life situation? The roles of 
appraisal and coping. Journal of Personality and Social Psychology, 68, 4, 687-695.

41. Dvir, T. y Shamir, B. (2003). Follower developmental characteristics as predicting transformational leadership: A longitudinal field study. The Leadership Quarterly, 14, 3, 327-344.

42. Dvir, T., Eden, D., Avolio, B.J. y Shamir, B. (2003). Impact of transformational leadership on follower development and performance: A field experiment. Academy of Management Journal, 45, 735-744.

43. Dekel, S., Ein-Dor, T. y Solomon, Z. (2012). Posttraumatic growth and posttraumatic distress: A longitudinal study. Psychological Trauma: Theory, Research, Practice, and Policy, 4, 1, 94-101.

44. Bliese, P.D. (2006). Social Climates: Drivers of soldier well-being and resilience. En A.B, Adler, C.A., Castro y T.W. Britt (Eds.). Military Life: The Psychology of Serving in Peace and Combat (Vol.2): Operational Stress. Westport, CT: Praeger Security International.

45. Adler, A.B., Bliese, P.D. y Castro, C.A. (2011). Deployment psychology: Evidence-based strategies to promote mental health in the military. Washington DC: American Psychological Association.

46. Nash, W.P., Steenkamp, M., Conoscenti, L. y Litz, B. (2011). The stress continuum model: a military organizational approach to resilience and recovery. En S.M. Southwick, B.T. Litz, D. Charney y M.J. Friedman (Eds.): Resilience and mental health: Challenges across the lifespan. New York: Cambridge University Press.

47. Laurence, J.H. y Matthews, M.D. (2012). The Oxford handbook of military psychology. New York: Oxford University Press.

48. Bardera, M.P. y Osca, A. (2009). Estrés laboral, autoconcepto y salud en una muestra de militares españoles. Sanidad Militar, 65, 3, 152-163.

49. Osca, A., González-Camino, G., Bardera, P. y Peiró, J.M. (2003). Estrés de rol y su influencia sobre el bienestar psíquico y físico en soldados profesionales. Psicothema, 15, 1, 54-57.

50. Castro, C.A. y Adler, A.B. (2011). Military mental health training: building resilience. En S.M. Southwick, B.T. Litz, D. Charney y M.J. Friedman (Eds.): Resilience and mental health: Challenges across the lifespan. New York: Cambridge University Press.

51. Mental Health Advisory Team 5. (2008, February 14). Mental Health Advisory Team (MHAT) V Operation Iraqi Freedom 06-08: Iraq, Operation Enduring Freedom 8: Afghanistan (Office of the Surgeon Multi-National Force- Iraq, Office of the Command Surgeon, Office of the Surgeon General United States Army Medical Command).

52. Bates, M.J., Bowles, S., Hammermeister, J., Stokes, C., Pinder, E., Moore, M., et al. (2010). Psychological fitness. Military Medicine, 15, 8:21.

53. Hobfoll, S.E. (1989). Conservation of resources: a new attempt at conceptualizing stress. American Psychologist, 44, 3, 513-24.

54. Demerouti, E., Bakker, A.B., Nachreiner, F., et al (2001). The job demandsresources model of burnout. Journal of Applied Psychology, 86, 499-512.

55. SCImago. (2007). SJR — SCImago Journal \& Country Rank. Retrieved December 18, 2012, from http://www.scimagojr.com

56. Giménez-Toledo, E., Tejada-Artigas, A. y Mañana-Rodríguez, J. (2012). Scholarly Publishers Indicators (SPI). Disponible en: http://epuc.cchs.csic.es/SPI

57. Gelkopf, M., et al. (2008). The Impact of 'Training the Trainers' Course for Helping Tsunami-Survivor Children on Sri Lankan Disaster Volunteer Workers. International Journal of Stress Management, 15(2): pp. 117-135.

58. Haglund, M.E.M., et al. (2007). Psychobiological Mechanisms of Resilience: Relevance to Prevention and Treatment of Stress-Related Psychopathology, Development and Psychopathology, 19: pp. 889-920.

59. Hjemdal, O., et al. (2006). Predicting Psychiatric Symptoms, Clinical Psychology and Psychotherapy, 13: pp. 194-201.

60. Southwick, S., et al., (2008). Adaptation to Stress and Psychobiological Mechanisms of Resilience. En Biobehavioral Resilience to Stress, B.J. Lukey and V. Tepe, Eds. CRC Press: Boca Raton, FL.

61. Tugade, M.M. y Fredrickson, B.L. (2004). Resilient Individuals Use Positive Emotions to Bounce Back from Negative Emotional Experiences, Journal of Personality and Social Psychology, 86(2): pp. 320-333.

62. Luskin, F., et al. (2002). A Controlled Pilot Study of Stress Management Training of Elderly Patients with Congestive Heart Failure, Preventive Cardiology, (Fall): pp. 168-17.

63. McCraty, R., et al., (1995). The Effects of Emotions on Short-Term Power Spectrum Analysis of Heart Rate Variability, American Journal of Cardiology, 76(4): pp. 1089-1093.
64. McCraty, R., Atkinson, M. y Tomasino, D. (2003). Impact of Workplace Stress Reduction Program on Blood Pressure and Emotional Health in Hypertensive Employees, Journal of Alternative and Complementary Medicine, 9(3): pp. 355-369.

65. Adler, A.B., Bliese, P.D., McGurk, D., Hoge, C.W. y Castro, C.A. (2011). Battlemind debriefing and battlemind training as early interventions with soldiers returning from Iraq: Randomization by platoon. Sport, Exercise, and Performance Psychology, 1, S, 66-83.

66. Affleck, G. y Tennen, H. (1996). Construing Benefits from Adversity: Adaptational Significance and Dispositional Underpinnings. Journal of Personality, 64(4): pp. 899-922.

67. Bilgin, M. (2009). Developing a cognitive flexibility scale: Validity and reliability studies. Social Behavior and Personality, 37(3):343-54.

68. Conger, R.D., Rueter, M.A. y Elder, G.H.J. (1999). Couple Resilience to Economic Pressure, Journal of Personality and Social Psychology, 76(1): pp. 54-71.

69. Chesney, MA, Neilands TB, Chambers DB, et al. (2006). A validity and reliability study of the coping self-efficacy scale. British Journal of Health Psychology, 11(3):421-37.

70. Williams, A., Hagerty, B.M., Yousha, S.M., Horrocks, J., Hoyle, K.S., y Liu, D. (2004). Psychosocial effects of the boot strap intervention in Navy recruits. Military Medicine, 169,10, 814-822

71. Jha AP, Krompinger J, Baime MJ. (2007). Mindfulness training modifies subsystems of attention. Cognitive Affective Behavioral Neuroscience. $7(2): 109-19$

72. Jha AP, Stanley EA, Kiyonaga A, et al. (2010). Examining the protective effects of mindfulness training on working memory capacity and affective experience. Emotion, 10(1):54-64.

73. Gillham, J.E., et al. (2007). School-Based Prevention of Depressive Symptoms: A Randomized Controlled Study of the Effectiveness and Specificity of the Penn Resilience Program. Journal of Consulting and Clinical Psycho$\log y, 75(1)$ : pp. 9-19.

74. McCraty, R., et al. (1999). The Impact of an Emotional Self-Management Skills Course on Psychosocial Functioning and Autonomic Recovery to Stress in Middle School Children. Integrative Physiological and Behavioral Science, 34(4): pp. 246-268.

75. McCraty, R., et al. (2009). New Hope for Correctional Officers: An Innovative Program for Reducing Stress and Health Risks. Applied Psychophysiology and Biofeedback, 34(4

76. Stanley, E.A., et al. (2012). Mind Fitness in Counterinsurgency: Addressing the Cognitive Requirements of Population-Centric Operations. Military Review, 90(1).

77. Maddi, S.R. (2007). Relevance of Hardiness Assessment and Training to the Military Context. Military Psychology, 19(1): pp. 61-70.

78. Watson, P. J., Ritchie, E.C., et al. (2006). Improving Resilience Trajectories Following Mass Violence and Disaster. En B.S., Ritchie, P.J. Watson y M.J. Friedman (Eds): Interventions Following Mass Violence and Disasters: Strategies for Mental Health Practice, New York: Guilford Press.

79. Conger, R.D. y Conger, K.J. (2002). Resilience in Midwestern Families: Selected Findings from the First Decade of a Prospective, Longitudinal Study. Journal of Marriage and Family, 64(2): pp. 361-373.

80. Fraley, R.C., et al. (2006). Attachment and Psychological Adaptation in High Exposure Survivors of the September 11th Attack on the World Trade Center. Personality and Social Psychology Bulletin, 32(4): pp. 538-551.

81. Hobfoll, S.E., et al. (2007). Five Essential Elements of Immediate and MidTerm Mass Trauma Intervention: Empirical Evidence. Psychiatry, 70(4): pp. 283-315.

82. Solomon, Z., Mikulincer, M. y Avitzur, E. (1988). Coping, Locus of Control, Social Support, and Combat-Related Posttraumatic Stress Disorder: A Prospective Study. Journal of Personality andSocial Psychology, 55(2): pp. 279-285.

83. Zach, S., Raviv, S. y Inbar, R. (2007). The Benefits of a Graduated Training Program for Security Officers on Physical Performance in Stressful Situations. International Journal of Stress Management, 14(4): pp. 350-369.

84. Hystad SW, Eid J, Johnsen BH, et al. (2010). Psychometric properties of the revised Norwegian dispositional resilience scale. Scandinavian Journal of Psychology.

85. Bartone PT. (1999). Hardiness protects against war-related stress in Army reserve forces. Consulting Psychology Journal. 51:72-82. 


\section{Revisión de programas de resiliencia basados en la evidencia en los ejércitos}

86. Bartone PT. (2007). Test-retest reliability of the Dispositional Resilience Scale -15, a brief hardiness scale. Psychological Reports, 101:943-4.

87. Eschleman, K.J., Bowling, N.A. y Alarcon, G.M. (2010). A meta-analytic examination of hardiness. International Journal of Stress Management, 17, 4, 277-307

88. Branscome, TA, Swoboda JC, Fatkin LT. (2007). An Initial Investigation of Factors Affecting Multi-Task Performance; ARL-TR-4025; U.S. Army Research Laboratory: Aberdeen Proving Ground, MD.

89. Fatkin LT, Hudgens GA. (1994). Stress Perceptions of Soldiers Participating in Training at the Chemical Defense Training Facility: The Mediating Effects of Motivation, Experience, and Confidence Level; ARL-TR-365; U.S. Army Research Laboratory: Aberdeen Proving Ground, MD.

90. Emmons, R. A., Cheung, C. y Tehrani, K. (1998). Assessing spirituality through personal goals: Implications for research on religion and subjective well-being. Social Indicators Research, 45, 391-422.

91. Mahoney, A., Pargament, K. I., Cole, B., Jewell, T., Magyar, G. M., Tarakeshwar, N., Phillips, R. (2005). A higher purpose: The sanctification of strivings in a community sample. International Journal for the Psychology of Religion, 15, 239-262.

92. Harris, J.I., Erbes, C.R., Engdahl, B.E., et al. (2008). Christian religious functioning and trauma outcomes. Journal of Clinical Psychology,64, 17-29.

93. Stouffer, S.A., Lumsdaine, A.A., Lumsdaine, M.H., Williams, R.W., Jr., Smith, M.B., Janis, I.L., ... Cotrell, L. S., Jr. (1949). The American soldier: Combat and its aftermath (Vol. 2). New York: Wiley.

94. Witvliet, C.V.O., Phillips, K.A., Feldman, M.E. y Beckham, J.C. (2004). Posttraumatic mental and physical health correlates of forgiveness and religious coping in military veterans. Journal of Traumatic Stress, 17, 269-273.

95. Fontana, A. y Rosenheck, R. (2004). Trauma, change in strength of religious faith, and mental health service use among veterans treated for PTSD. Journal of Nervous and Mental Disease, 192, 579-584.

96. Benda, B. B., DiBlasio, F. A. y Pope, S. K. (2006). Spiritual well-being, relationships, and work satisfaction in the treatment of homeless veterans with alcohol and other drug problems. Alcoholism Treatment Quarterly, 24, 109-124

97. Ano, G.G. y Vasconcelles, E.B. (2005). Religious Coping and Psychological Adjustment to Stress: A Meta-Analysis, Journal of Clinical Psychology, 61(4): pp. 461-480.

98. Donahue, M. J. (1985). Intrinsic and extrinsic religiousness: Review and meta-analysis. Journal of Personality and Social Psychology, 48, 400-419.

99. Palmer, C. (2008). A Theory of Risk and Resilience Factors in Military Families, Military Psychology, 20(3): pp. 205-217.

100. Ritchie, E.C., Watson, P.J. y Friedman, M.J. (Eds): Interventions Following Mass Violence and Disasters: Strategies for Mental Health Practic (2006). New York: Guilford Press.

101. Meichenbaum, D. (1985). Stress inoculation training. New York: Pergamon Press.

102. Alvarez, J. y Hunt, M. (2005). Risk and resilience in canine search and rescue handlers after 9/11. Journal of Traumatic Stress, 18, 497-505.

103. Hagh-Shenas, H., Goodarzi, M.A., Dehbozorgi, G. y Farashbandi, H (2005). Psychological consequences of the Bam earthquake on professional and nonprofessional helpers. Journal of Traumatic Stress, 18, 477-483.

104. Flaxman, P.E. y Bond, F.W. (2010). A randomised workside comparison of acceptance and commitment therapy and stress inoculation training. Behaviour Research and Therapy, 48: 816-820.

105. Hammerfald, K., Eberle, C., Grau, M., Kinsperger, A., Zimmermann, A., Ehlert, U. Y Gaab, J. (2006). Persistent effects of cognitive-behavioral stress management on cortisol responses to acute stress in healthy subjects: A randomized controlled trial. Psychoneuroendocrinology, 31, 333-339.

106. Rose, R. D., Buckey, J. C., Zbozinek, T. D., Motivala, S. J., Glenn, D. E., Cartreine, J. A. y Craske, M. G. (2013). A randomized controlled trial of a self-guided, multimedia, stress management and resilience training program. Behaviour Research and Therapy, 51(2), 106-112.

107. Cornum, R.L. y Lester, P.B. (2012). Comprehensive Soldier Fitness: Why? And Why Now? En J.H. Laurence y M.D. Matthews (Eds.), The Oxford Handbook of Military Psychology. New York: Oxford University Press.

108. Black, K. y Lobo, M. (2008). A Conceptual Review of Family Resilience Factors. Journal of Family Nursing, 14(1): pp. 33-55.

109. Coleman, M. y Ganong, L. (2002). Resilience and Families, Family Relations, 51(2): pp. 101-102.
110. Letourneau, N., et al., (2001). Supporting Parents: Can Intervention Improve Parent-Child Relationships? Journal of Family Nursing, 7(2): pp. 159-187.

111. Castro, C.A. y McGurk, D. (2007). The Intensity of Combat and Behavioral Health Status, Traumatology, 13(4): pp. 6-23.

112. Campbell, D., Campbell, K. y Ness, J. (2008). Resilience Through Leadership. En B.J. Lukey y V. Tepe (Eds.) Biobehavioral Resilience to Stress, CRC Press: Boca Raton, FL.

113. Brailey, K., et al., (2007). PTSD Symptoms, Life Events, and Unit Cohesion in U.S. Soldiers: Baseline Findings from the Neurocognition Deployment Health Study, Journal of Traumatic Stress, 20(4): pp. 495-503.

114. Eid, J. y Johnsen, B.H. (2002). Acute Stress Reactions After Submarine Accidents, Military Medicine, 167(5): pp. 427-431.

115. Butler, L.D., Morland, L.A. y Leskin, G.A. (2007). Psychological Resilience in the Face of Terrorism. En Bonger et al. (Eds): Psychology of Terrorism. Oxford University Press: Oxford, UK

116. Seligman, M.E.P., Steen, T.A., Park, N. y Peterson, Ch. (2005). Positive psychology progress: Empirical validation of interventions. American Psychologist, 60, 5, 410- 421.

117. Adler, A.B., Castro, C.A. y McGurk., D. (2009). Time-driven Battlemind Psychological debriefing: A group-level early intervention in combat. Military Medicine, 174, 22-28.

118. Hoge, C.W., Castro, C.A., Messer, S.C., McGurk, D., Cotting, D.I. y Koffman, R.L. (2004). Combat duty in Iraq and Afghanistan, menta health problems, and barriers to care. New England Journal of Medicine, 351, 13-22.

119. Greenberg, N. y Jones, N. (2011). Optimizing mental Health support in the military: The role of peers and leaders. En A.B. Adler, P.D. Bliese y C.A. Castro (Eds.): Deployment Psychology. Washington DC: American Psychological Association.

120. Castro, C.A., Hoge, C.W. y Cox, A.L. (2006). Battlemind Training: Building Soldier Resiliency. En Human Dimensions in Military Operations- Military Leaders' Strategies for Addressing Stress and Psychologogical Support (pp. 42-1 - 42-6). Meeting procedings RTO-MP-HFM-134, Paper 42. Neuillysur-Seine, France: RTO.

121. Bisson, J.I., Jenkins, P.L., Alexander, J. y Bannister, C. (1997). Randomized controlled trial of psychological debriefing for victims of acute burn trauma. British Journal of Psychiatry, 171, 78- 81.

122. Hobbs, M., Mayou, R., Harrison, B. y Worlock, P. (1996). A randomized controlled trial of psychological debriefing for victims of road traffic accidents. British Medical Journal, 313, 1438- 1439.

123. Rose, S., Brewin, C.R., Andrews, B. y Kirk, M. (1999). A randomized controlled trial of individual psychological debriefing for victims of violent crime. Psychological Medicine, 29, 793- 799.

124. Sijbrandij, M., Olff, M., Reitsma, J.B., Carlier, I.V.E. y Gersons, B.P.R. (2006). Emotional or educational debriefing after psychological trauma: Randomised controlled trial. British Journal of Psychiatry, 189, 150- 155.

125. Adler, A.B., Litz, B.T., Castro, C.A., Suvak, M., Thomas, J.L., Burrell, L, et al. (2008). Group randomized trial of critical incident stress debriefing provided to U.S. peacekeepers. Journal of Traumatic Stress, 21, 253-263.

126. Brunwasser, S.M., Gillham, J.E. y Kim, E.S. (2009). A meta-analytic review of the Penn Resiliency Program's effect on Depressive Symptoms. Journal of Consulting and Clinical Psychology, 77, 6, 1042-1054.

127. Seligman, M.E.P. (2011). Helping american soldiers in time of war: Reply to comments on the Comprehensive Soldier Fitness special issue. American Psychologist, 66, 7, 646-647.

128. Reivich, K.J., Seligman, M.E.P. y McBride, S. (2011). Master Resilience Training in the U.S. Army. American Psychologist, 66, 1, 25-34.

129. Peterson, C., Park, N. y Castro, C.A. (2011). Assessment for the U.S. Army Comprehensive Soldier Fitness Program: The Global Assessment Tool. American Psychologist, 66, 1, 10-18.

130. Lester, P.B., McBride, S., Bliese, P.D. y Adler, A.B. (2011). Bringing science to bear: An empirical assessment of the Comprehensive Soldier Fitness Program. American Psychologist, 66, 1, 77-81.

131. Peterson, C., Park, N. y Castro, C.A. (2011). Assessment for the U.S. Army Comprehensive Soldier Fitness Program: The Global Assessment Tool. American Psychologist, 66, 1, 10-18.

132. DiClemente, C.C., Marinilli, A.S., Singh, M. y Bellino, L.E. (2001). The role of feedback in the process of health behavior change. American Journal of Health Behavior, 25, 217-227. 
133. Prochaska, J.O., DiClemente, C.C. y Norcross, J.C. (1992). In search of how people change: Applications to addictive behaviors. American Psychologist, 47, 1102-114.

134. Masten, A.S. y Reed, M.G.J. (2002). Resilience in development. En S.J. Lopez y C.R. Snyder (Eds.). Oxford Handbook of Positive Psychology. (2 $\left.{ }^{\mathrm{a}} \mathrm{Ed}\right)$. New York: Oxford University Press.

135. Ellis, A. (1962). Reason and emotion in psychotherapy. New York: Lyle Stuart.

136. Goffman, E. (1963). Stigma: Notes on the management of spoiled identity. NJ: Prentice Hall.

137. Lester, P. B., Harms, P. D., Bulling, D. J., Herian, M. N. y Spain, S. M. (2011a, February). Evaluation of relationships between reported resilience and soldier outcomes: Report \#1: Negative outcomes (suicide, drug use, and violent crime) (Tech. Rep. 1).
138. Lester, P. B., Harms, P. D., Bulling, D. J., Herian, M. N. y Spain, S. M. (2011b, April). Evaluation of relationships between reported resilience and soldier outcomes: Report \#2: Positive performance outcomes in officers (promotions, selections, and professions). (Tech. Rep. 2).

139. Lester, P.B., Harms, P.D., Herian, M.N., Krasikova, D.V. y Beal, S.J. (2011, Diciembre). The Comprehensive Soldier Fitness program evaluation: Report \#3: Longitudinal Analysis of the impact of Master Resilience Training on self-reported resilience and psychological health data. (Tech. Rep. 3).

140. Harms, P.D., Herian, M.N., Krasikova, D.V., Vanhove, A. y Lester, P.B. (2013). The comprehensive soldier and family fitness program evaluation report \# 4: Evaluation of resilience training and mental and behavioral health outcomes. Monterrey, CA: Office of the Deputy Under Secretary of the Army. 